\title{
EEG Source Reconstruction Performance as a Function of Skull Conductance Contrast
}

\author{
Hansen, Sofie Therese; Hansen, Lars Kai
}

Published in:

Proceedings of the 40th IEEE International Conference on Acoustics, Speech and Signal Processing (ICASSP 2015)

Link to article, DOI:

10.1109/ICASSP.2015.7178085

Publication date:

2015

Document Version

Peer reviewed version

Link back to DTU Orbit

Citation (APA):

Hansen, S. T., \& Hansen, L. K. (2015). EEG Source Reconstruction Performance as a Function of Skull Conductance Contrast. In Proceedings of the 40th IEEE International Conference on Acoustics, Speech and Signal Processing (ICASSP 2015) (pp. 827-831). IEEE. https://doi.org/10.1109/ICASSP.2015.7178085

\section{General rights}

Copyright and moral rights for the publications made accessible in the public portal are retained by the authors and/or other copyright owners and it is a condition of accessing publications that users recognise and abide by the legal requirements associated with these rights.

- Users may download and print one copy of any publication from the public portal for the purpose of private study or research.

- You may not further distribute the material or use it for any profit-making activity or commercial gain

- You may freely distribute the URL identifying the publication in the public portal 


\title{
EEG SOURCE RECONSTRUCTION PERFORMANCE AS A FUNCTION OF SKULL CONDUCTANCE CONTRAST
}

\author{
Sofie Therese Hansen, Lars Kai Hansen
}

Technical University of Denmark; Department of Applied Mathematics and Computer Science; DK-2800 Kgs. Lyngby

\begin{abstract}
Through simulated EEG we investigate the effect of the forward model's applied skull:scalp conductivity ratio on the source reconstruction performance. We show that having a higher conductivity ratio generally leads to improvement of the solution. Additionally we see a clear connection between higher conductivity ratios and lower coherence, thus a reduction of the ill-posedness of the EEG inverse problem. Finally we show on real EEG data the stability of the strongest source recovered across conductivity ratios.
\end{abstract}

Index Terms - EEG source reconstruction, Inverse problem, Forward models, Sparsity, Variational approximation.

\section{INTRODUCTION}

The promise of electroencephalography (EEG) based brain imaging is high in settings where real-time and quasi-natural conditions are required [1]. These situations call for a neuroimaging technique with high temporal resolution and equipment which is portable and lightweight [2]. The span of applications for EEG source reconstruction includes clinical deployment where EEG is used in, e.g., diagnosis support [3, 4], home use for self-monitoring [2], and cognitive neuroscience brain imaging for exploring neural dynamics $[1,5,6]$.

The EEG inverse problem is straightforward to formulate based on the linear relation between EEG sensors and generators provided by the quasi-static approximation of Maxwell's equations [1]. However, the problem is highly underdetermined; electrodes are counted in hundreds but potential sources in thousands. The solution is therefore non-trivial and research in the area is extensive [7, 8, 9, 10, 11]. Several studies have shown that invoking multiple measurement vector (MMV) models by assuming common support across time improve source recovery $[8,9,10]$. Also beneficial is complexity control obtained by enforcing sparsity; warranted by the assumption that the activity of interest is focal and by the dipolar nature of independent source components [10, 12].

The forward models (relating the EEG sources to the electrodes) are inherently very coherent and as explained in [13] this adds to the ill-posedness of the inverse problem. Often the forward model is assumed fixed and known. The boundary element method (BEM) is an efficient way of obtaining a

This work is supported by the Stibo-Foundation and the Danish Lundbeck Foundation via the Center for Integrated Molecular Brain Imaging (CIMBI). forward model where the geometry of the layers between the electrodes and sources can be included [1]. For instance, the three-layered BEM models the scalp, skull and cortex, with information from either structural head scans or through template models [1]. The used conductivity ratios of these layers have been shown to affect the ability to correctly localize the EEG sources [14]. Especially the conductance of the skull is important and due to its complex structure the conductivity differs not only between subjects but also within a single subject [15]. Plis et al. suggest to account for the uncertainty of the skull conductance by including uncertainty in the source location estimate [15]. In [16] Lew et al. propose to include the skull conductivity as a parameter to model.

In [17] Wang et al. compare the source localization error obtainable with skull:scalp conductivity ratios 1/15, 1/20, $1 / 25$ and $1 / 80$ using both the data generating ratio and the three incorrect ratios. With a signal to noise ratio (SNR) of $10 \mathrm{~dB}$ and one planted source, an error of $9 \mathrm{~mm}$ is reported when using the true ratio. Single equivalent current dipole was used for source localization and the solution is thus biased by the knowledge of the number of planted sources.

The basic question of this paper is how does the skull conductance contrast affect source reconstruction performance?. We will answer this by systematically investigating the performance of the source reconstruction as a function of the skull conductivity with several state of the art inverse problem solvers. Rather than considering the error introduced when using a wrong conductance ratio as in $[15,16]$, we use the same ratio for generating the simulations as we use in the reconstruction. We thus demonstrate across 100 ratios the inherent variability of the reconstruction performance. For one of the solvers we also show that a simple cross-validation based optimization of hyperparameters leads to close to oracle performance for a large range of conductivities; we thus expand on the method used in [17]. This insight we use to investigate, in real EEG data, the stability of the recovered sources with respect to the applied conductivity ratio.

\section{MATERIALS AND METHODS}

\subsection{Neuroimaging data}

Structural magnetic resonance imaging (sMRI) scans from a subject participating in a multimodal study exploring the neural mechanisms of face perception were acquired 

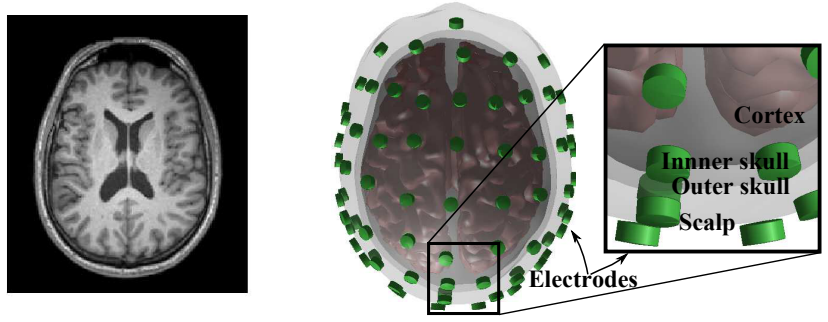

Fig. 1. MRI scan of a subject (Left) and the segmentation into scalp, skull and cortex (Right). The 70 electrodes are placed in the standard 10-20 system.

through the courtesy of Henson and Wakeman [18]; available at //ftp.mrc-cbu.cam.ac.uk/personal/rik. henson/wakemandg_hensonrn/. The T1 weighted images were recorded on a Siemens 3T Trio. We use the EEG data, recorded with 70 10-20 arranged $\mathrm{Ag}-\mathrm{AgCl}$ electrodes, to create the average difference between seeing faces and scrambled faces. See stimulus design of the study in [5].

Preprocessing is done using MATLAB (MathWorks Inc.) in part by scripts provided by Wakeman and Henson. Segmentation of the subject's sMRI is done using SPM8 [19] and co-registration to the EEG electrodes is obtained through fiducials placed on the nasion and the left and right pre-auricular, together with headshape points. The cortex mesh is set to consist of 8196 vertices. The BEM, in the "bemcp" implementation (by Phillips [20]), is used to create 100 forward models having the conductivities $[1, c, 1] \cdot 0.33$ of brain:skull:scalp, where 100 samples of $c$ are drawn from a uniform distribution between $1 / 250$ and $1 / 15$ (in SPM8 default setting is $1 / 80$ ). The constructed layers can be seen in Fig. 1 together with the electrodes.

\subsection{Synthetic EEG data}

Five synthetic data sets are constructed by planting two active sources; for all configurations one source is placed in each hemisphere (see Fig. 4). The sources are given the temporal dynamics of two synchronous sinusoids across 25 time samples. The synthetic source distributions are projected to the 70 electrodes through each of the 100 forward models. Additionally white noise is added to yield $\mathrm{SNR}=5 \mathrm{~dB}$ in ten repetitions (in total $5 \times 100 \times 10=5000$ data sets).

\subsection{Source reconstruction methods}

We will apply two source reconstruction methods to investigate the generated forward models; MFOCUSS [8] and our adapted version of the variational garrote (VG) [21]. The methods are similar in three ways. 1) They both have a regularization parameter that controls the density of the solution. 2) They assume common support across time samples. 3) Their relation to the $\ell_{0}$-norm solution.
We use the implementation of MFOCUSS as provided by Zhang; http://dsp.ucsd.edu/ zhilin/TMSBL. html. MFOCUSS by Cotter et al. [8] is an MMV version of FOCUSS (FOCal Underdetermined System Solution), which finds a reweighted minimum norm (MN) solution [7, 22], and thus approximates the $\ell_{0}$ (numerousity) regularizer [10]. FOCUSS is initialized with the MN solution and then iteratively increases large solution values and decreases small [7].

The regularization parameter of MFOCUSS is the noise level of the data. This parameter is in the experiments varied from $10^{-11}$ to 1 in 100 steps on the $\log 10$ scale. We use MFOCUSS in an oracle like setup where we report the solution with lowest localization error and highest F-measure across investigated noise levels. This is of course not applicable to real data where we would not have ground truth available. However on the synthetic data we want to use this approach to find the best possible solution obtainable using MFOCUSS and a forward model with a specific skull:scalp conductivity ratio. The localization error is the average Euclidean distance between the two planted and two estimated strongest sources. And F-measure $=2 \cdot \mathrm{TP} /(\mathrm{P}+\mathrm{TP}+\mathrm{FP})$ [23]; TP, FP and P are the number of true, false and all actual positives, respectively. Perfect localization yields an F-measure of 1 .

The second source reconstruction method we test is our MMV modified version of VG [21]. The $\ell_{0}$ regularization is achieved in VG for the single measurement by including a binary variable $s_{n} \in\{0,1\}$ (for electrode $n=1 . . N$ ) in the linear relation between electrodes and sources, modelling whether a source is active or not. In our modification, detailed in [24], we extend VG to MMV by simply fixing this binary variable across time; we call it the time-expanded VG (teVG). The binary variable has the prior $p(\mathbf{s} \mid \gamma)=\prod_{n=1}^{N} p\left(s_{n} \mid \gamma\right)$ where $p\left(s_{n} \mid \gamma\right)=\frac{\exp \left(\gamma s_{n}\right)}{1+\exp (\gamma)}$ [21]. The hyperparameter $\gamma$ is thus sparsity controlling.

The solution scheme proposed by Kappen et al. is based on Bayesian inference by maximizing the posterior probability. As this is non-trivial, variational approximation is employed. First a marginalization over $\mathbf{s}$ is performed and $q(\mathbf{s})=\prod_{n=1}^{N} q_{n}\left(s_{n}\right)$ is introduced, where $q_{n}\left(s_{n}\right)=m_{n} s_{n}+\left(1-m_{n}\right)\left(1-s_{n}\right)$ [21]. The parameter $m_{n}$ is the variational mean and can be interpreted as the probability of $s_{n}$ being active, thus it has values between 0 and 1. Next, Jensen's inequality is applied giving us the lower bound; i.e. the free energy $F$. We pose the free energy in a dual formulation following [21], and minimize it by setting the partial derivatives equal to zero, except for $\gamma$ which we estimate through cross-validation. In Fig. 2 we show how we split the 70 electrodes into four folds. The partitioning is done with the aim of maximizing the spread of the 17-18 electrodes contained in each fold.

Kappen et al. suggest to obtain the solution through fixed point iteration, which has a computational complexity that scales quadratic in the number of electrodes and linearly in 


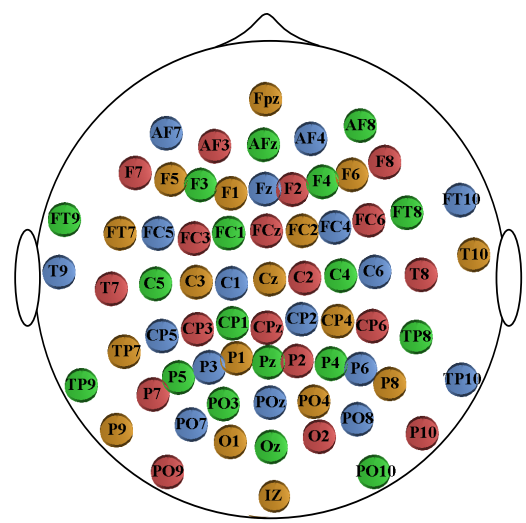

Fig. 2. Partioning of the 70 10-20 arranged EEG electrodes into four folds represented by the coloring.

the number of sources, thus keeping computation time low (further reduced using common support in teVG). As the VG solves a non-convex problem a smoothing parameter is needed to control the variational mean updates. How to set this parameter is non-trivial, therefore we suggest to control the updates of $\mathbf{m}$ through gradient descent. The update to $\mathbf{m}$ will now be $\mathbf{m}-\eta \frac{\partial F}{\partial \mathbf{m}}$. The parameter $\eta$ now needs to be set, however the solution is much less sensitive to this parameter, than the before mentioned smoothing parameter. A starting value of $\eta_{0}=10^{-3}$ is heuristically set. At each iteration the decrease or increase in the free energy will increase the $\eta$-value by $10 \%$ or reduce it by $50 \%$, respectively. Additional convergence speed is gained by raising $\eta$ to $\eta_{0}$ for a number of iterations. We call this faster VG version GDteVG (Gradient Descent time-expanded Variational Garrote).

We apply GDteVG in two settings. On the synthetic data we 1) run GDteVG in the same oracle setup as MFOCUSS, and 2) using cross-validation to find the regularization parameter, $\gamma$. On the real EEG data we can of course only do the latter. In the oracle setup we explore solutions with sparsity levels from -250 to -1 in 100 steps, and we cross-validate 25 steps of sparsity between -150 and -10 .

\section{RESULTS}

We investigate the matrix properties of the forward models through their coherence and condition number. The coherences of the forward models are shown in Fig. 3. As the forward models are very coherent we show ' 1 - the coherence'. Another matrix characteristic, the condition number, is high for these models (between $1.4 \cdot 10^{15}$ and $2.9 \cdot 10^{15}$ ) but is not found to be linked to the conductivity ratio.

The ability of the forward models to recover the sources they themselves have projected to the electrodes is now tested. We compare the forward models through localization error and F-measure in Fig. 4. MFOCUSS and GDteVG are run in the oracle setup described earlier and GDteVGcross is GDteVG with cross-validation on the regularization pa-

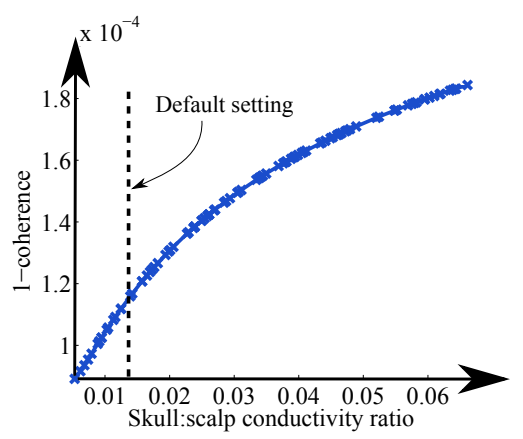

Fig. 3. ' $1-$ the coherence' of the 100 forward models.

rameter. There is a general trend that forward models with lower contrast, i.e., lower conductivity ratios, perform worse (source configuration 1, 2, 3 and in part 4). In the the fifth source configuration we see relatively limited effects of the contrast. Generally the GDteVG solver shows the lowest localization error and highest F-measure, and there is a good correspondence between the oracle and the cross-validation forms of the method, which allows us to make inferences for the real EEG data where ground truth is unavailable.

Finally we investigate the real EEG data described in section 2.1. As we now do not know the ground truth and can not report localization error, we instead track the strongest source found as a function of conductivity ratio, see Fig. 5. Across the 100 conductivity ratios five different sources dominate the source reconstruction solutions. Generally it is agreed that the activity happens posteriorly and 87 of the tested conductivity ratios place the strongest source in the right posterior inferior temporal lobe (red and green in Fig. 5). The solution is rather robust with respect to the time of maximum activity; having a mean of $154.4 \mathrm{~ms}$ with the standard deviation $1.5 \mathrm{~ms}$.

\section{CONCLUSION}

This contribution relies on advances in both EEG forward modeling $[14,15,16,17,20]$ and inverse inference techniques $[7,8,9,10,11,12,21,22]$ to expand on the dependence of skull conductivity on the source reconstruction performance.

For establishing an accurate electrical forward model for EEG, the conductivity distribution is needed. Here we have investigated the sensitivity of the solution to one important aspect herein, namely the skull conductivity contrast. We found that increasing the skull conductivity contrast reduces the forward model coherence, hence, in this aspect reduces the ill-posedness of the linear inverse problem. This relation was confirmed in experiments using two different sparse inverse problem solvers MFOCUSS and the variational garrote (VG). Both showed a higher localization error for low contrasts. We found that a simple cross-validation scheme could reliably be used to infer the sparsity level in VG and using the cross-validation scheme we showed that the most active dipoles found in a real EEG data set was relatively stable to the skull conductivity contrast for a wide window of values. 

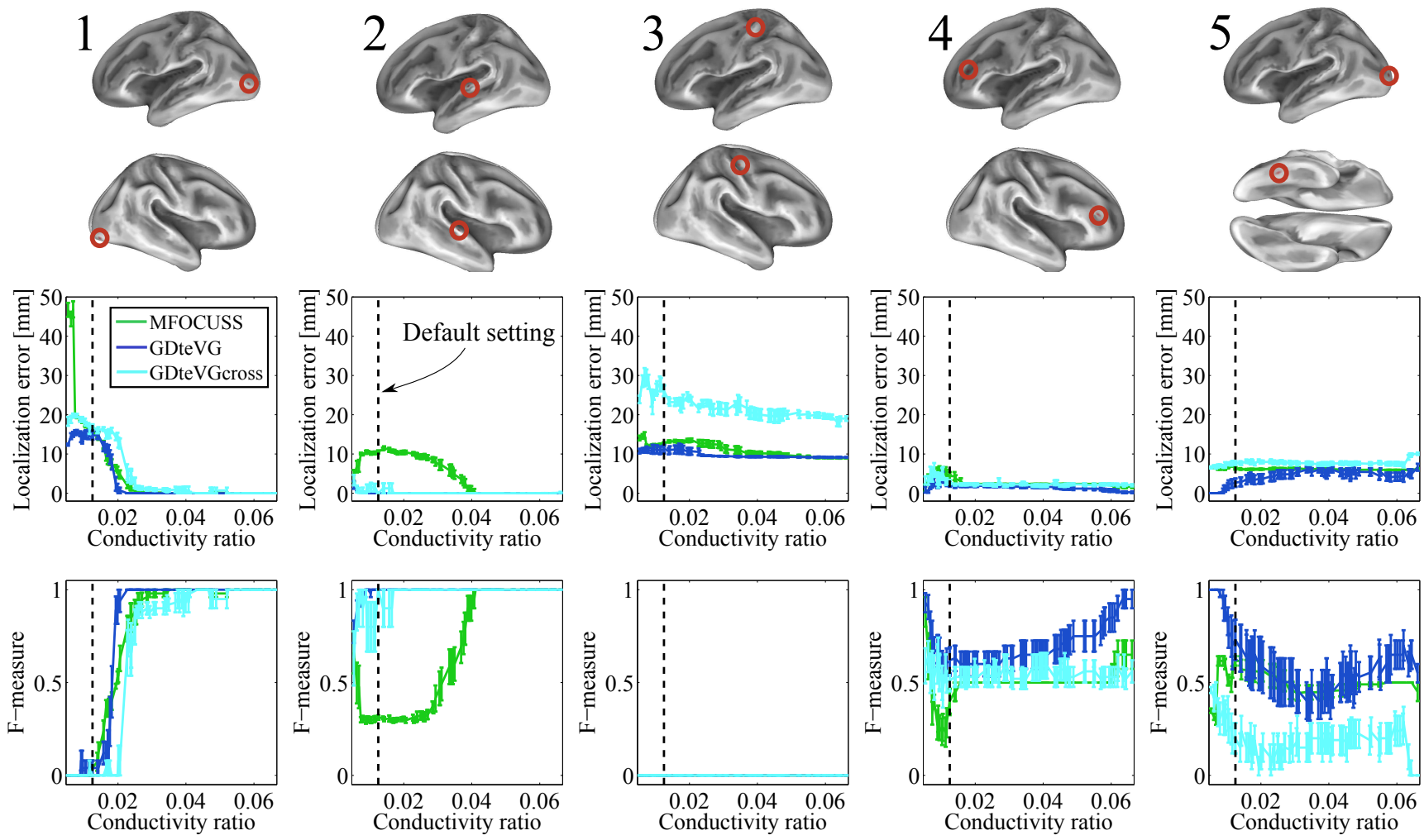

Fig. 4. Performance of MFOCUSS, GDteVG and GDteVGcross with forward models of varying skull:scalp conductivity ratios on synthetic data. The two former are favored by here reporting the lowest localization error (middle panel) and highest Fmeasure (lower panel) across regularization. At each conductivity ratio the investigated forward model is used as the data generating design matrix. The SPM8 default setting of the ratio is indicated by the dashed lines. Results are from five source configurations, each having two active sources; the locations of these can be seen in the top panel. Noise is added to give an SNR of $5 \mathrm{~dB}$, this is done in ten repetitions. In the implementations with MFOCUSS the columns of the forward models are scaled to unit norm while they are merely centered for VG.

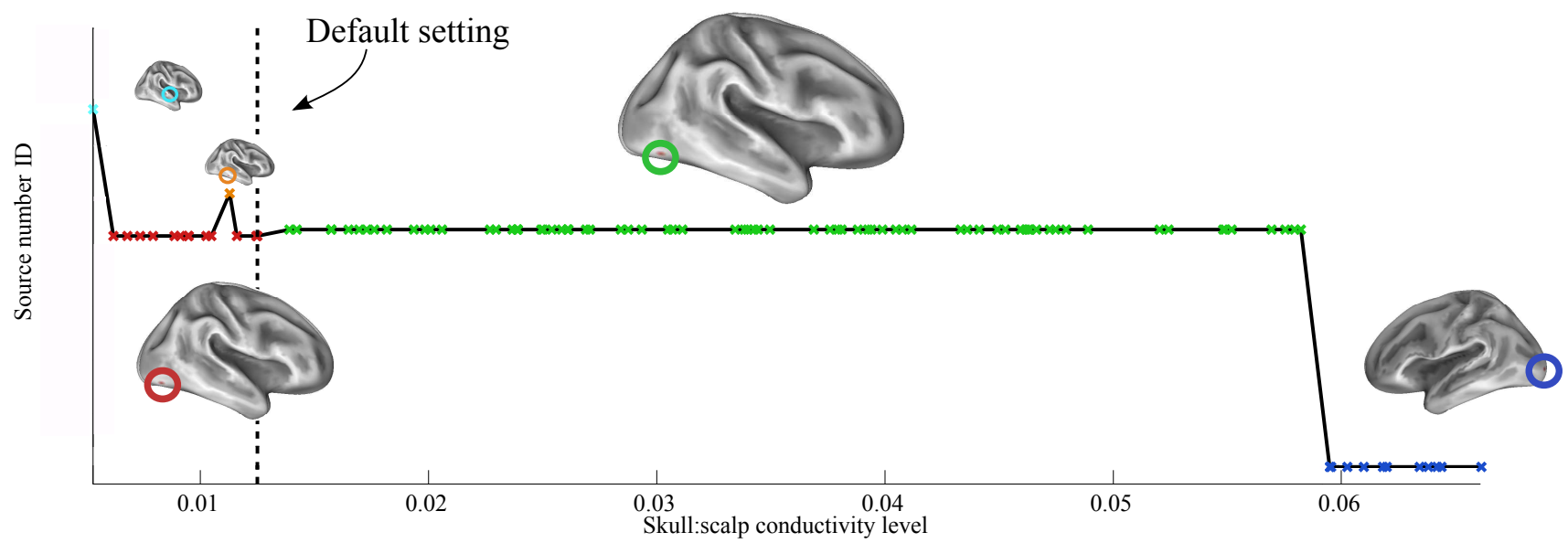

Fig. 5. Stability of the solution when using real EEG data across conductivity ratios (the SPM8 default setting is indicated). Each color represents a different dominating source, in total five unique strongest sources are found. Source reconstruction is performed using GDteVG with four-fold cross-validation. The EEG data is the differential signal of the conditions faces and scrambled faces. The signal is 23 time samples long, corresponding to $100 \mathrm{~ms}$, and begins $100 \mathrm{~ms}$ after stimuli. 


\section{REFERENCES}

[1] S. Baillet, J. C. Mosher, and R. M. Leahy, "Electromagnetic brain mapping," Signal Processing Magazine, IEEE, vol. 18, no. 6, pp. 14-30, 2001.

[2] A. Stopczynski, C. Stahlhut, J. E. Larsen, M. K. Petersen, and L. K. Hansen, "The smartphone brain scanner: A portable real-time neuroimaging system," PloS one, vol. 9, no. 2, pp. e86733, 2014.

[3] M. Teplan, "Fundamentals of EEG measurement," Measurement science review, vol. 2, no. 2, pp. 1-11, 2002.

[4] S. Hulbert and H. Adeli, "EEG/MEG-and imagingbased diagnosis of Alzheimers disease," Reviews in the neurosciences, vol. 24, no. 6, pp. 563-576, 2013.

[5] R. N. Henson, Y. Goshen-Gottstein, T. Ganel, L. J. Otten, A. Quayle, and M. D. Rugg, "Electrophysiological and haemodynamic correlates of face perception, recognition and priming," Cerebral cortex, vol. 13, no. 7, pp. 793-805, 2003.

[6] S. Kouider, C. Stahlhut, S. V. Gelskov, L. S. Barbosa, M. Dutat, V. De Gardelle, A. Christophe, S. Dehaene, and G. Dehaene-Lambertz, "A neural marker of perceptual consciousness in infants," Science, vol. 340, no. 6130, pp. 376-380, 2013.

[7] I. Gorodnitsky, J. George, and B. Rao, "Neuromagnetic source imaging with FOCUSS: a recursive weighted minimum norm algorithm," Electroencephalography and clinical Neurophysiology, vol. 95, pp. 231-251, 1995.

[8] S. F. Cotter, B. D. Rao, K. Engan, and K. Kreutzdelgado, "Sparse Solutions to Linear Inverse Problems with Multiple Measurement Vectors," IEEE Transactions on Signal Processing, vol. 53, no. 7, pp. 24772488, 2005.

[9] Z. Zhang and B. D. Rao, "Sparse signal recovery with temporally correlated source vectors using sparse Bayesian learning," Selected Topics in Signal Processing, IEEE, vol. 5, no. 5, pp. 912-926, 2011.

[10] A. Gramfort, M. Kowalski, and M. Hämäläinen, "Mixed-norm estimates for the M/EEG inverse problem using accelerated gradient methods," Physics in medicine and biology, vol. 57, no. 7, pp. 1937-61, 2012.

[11] C. Stahlhut, H. T. Attias, K. Sekihara, and D. Wipf, "A hier-archical Bayesian M/EEG imaging method correcting for incomplete spatio-temporal priors," 10th IEEE International Symposium on Biomedical Imaging (ISBI 2013, 2013.
[12] A. Delorme, J. Palmer, J. Onton, R. Oostenveld, and S. Makeig, "Independent EEG sources are dipolar," PloS one, vol. 7, no. 2, pp. 1-14, 2012.

[13] D. L. Donoho, M. Elad, and V. N. Temlyakov, "Stable recovery of sparse overcomplete representations in the presence of noise," Transactions on Information Theory, IEEE, vol. 52, no. 1, pp. 6-18, 2006.

[14] Z. Akalin Acar and S. Makeig, "Effects of forward model errors on EEG source localization," Brain topography, vol. 26, no. 3, pp. 378-396, 2013.

[15] S. M. Plis, J. S. George, S. C. Jun, D. M. Ranken, P. L. Volegov, and D. M. Schmidt, "Probabilistic forward model for electroencephalography source analysis., volume $=52$, year $=2007$, , Physics in medicine and biology, , no. 17, pp. 5309-5327.

[16] S. Lew, C. Wolters, a. Anwander, S. Makeig, and R. MacLeod, "Low resolution conductivity estimation to improve source localization," International Congress Series, vol. 1300, pp. 149-152, 2007.

[17] G. Wang and D. Ren, "Effect of brain-to-skull conductivity ratio on EEG source localization accuracy," BioMed research international, vol. 2013.

[18] R. N. Henson, D. G. Wakeman, V. Litvak, and K. J. Friston, "A Parametric Empirical Bayesian Framework for the EEG/MEG Inverse Problem: Generative Models for Multi-Subject and Multi-Modal Integration," Frontiers in human neuroscience, vol. 5, pp. 76:1-16, 2011.

[19] J. Ashburner, C.-C. Chen, R. Moran, R. N. Henson, V. Glauche, and C. Phillips, "SPM8 manual," Tech. Rep., The FIL Methods Group, 2012.

[20] C. Phillips, Source estimation in EEG: Combining anatomical and functional constraints, Ph.D. thesis, 2000.

[21] H. J. Kappen and V. Gómez, "The Variational Garrote," Machine Learning, pp. 1-26, 2013.

[22] I. Gorodnitsky and B. Rao, "Sparse signal reconstruction from limited data using FOCUSS: a re-weighted minimum norm algorithm," IEEE Transactions on Signal Processing, vol. 45, no. 3, pp. 600-616, 1997.

[23] J. Makhoul, F. Kubala, R. Schwartz, and R. Weischedel, "Performance measures for information extraction," in Proceedings of DARPA Broadcast News Workshop, pp. 249-252. Morgan Kaufmann Pub, 1999.

[24] S. T. Hansen, C. Stahlhut, and L. K. Hansen, "Expansion of the Variational Garrote to a Multiple Measurement Vectors Model," in Twelfth Scandinavian Conference on Artificial Intelligence, 2013, pp. 105-114. 